\title{
Las distorsiones cognitivas y el riesgo de suicidio en una muestra de adolescentes chilenos y colombianos: un estudio descriptivo-correlacional
}

\author{
Cognitive distortions and risk for suicidal behaviors, \\ considering Chilean and Colombian adolescents as sample: \\ A descriptive-correlational study is conducted
}

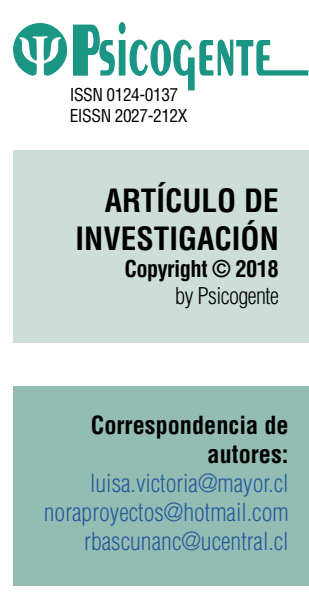

Recibido: $20-10-17$ Aceptado: $08-06-18$ Publicado: 08-01-19

\author{
Luisa Victoria Álvarez (iD) - Nora Yamilet Ayala Moreno (iD \\ Universidad Cooperativa de Colombia, Cali, Colombia \\ Ricardo Bascuñán Cisternas iD \\ Universidad Central de Chile, Santiago de Chile, Chile
}

Resumen

Objetivo: Determinar la relación entre las distorsiones cognitivas y el riesgo de suicidio en una muestra de adolescentes chilenos y colombianos.

Método: Este es un estudio descriptivo correlacional, de corte no experimental y transversal. El riesgo de suicidio se estableció con la Escala de Suicidalidad de Okasha y se identificaron las distorsiones cognitivas mediante el Inventario de Pensamientos Automáticos de Ruiz y Lujan. Ambas pruebas se realizaron en una muestra de adolescentes chilenos ( $n=200$; Colegio Alma Mater) y colombianos ( $n=200$; Institución Educativa Santa Librada) entre los 13 y 18 años de edad. Se seleccionó la muestra mediante un muestreo no probabilístico e intencional, y los participantes se separaron en grupo con riesgo de suicidio y grupo sin riesgo de suicidio (control). Se realizó un análisis descriptivo de la muestra, y una comparación de las distorsiones cognitivas entre los grupos. Finalmente, el análisis inferencial de los datos identificó la relación e intensidad entre distorsiones cognitivas y riesgo de suicidio.

Resultados: Se encontró relación estadísticamente significativa entre las distorsiones cognitivas y riesgo de suicidio, y las siguientes distorsiones presentaron la mayor intensidad en chilenos y colombianos: Filtraje, Interpretación del Pensamiento, Pensamiento Polarizado y Sobregeneralización. La correlación entre los resultados de adolescentes chilenos y colombianos es positiva y alta.

Conclusiones: Los resultados del presente estudio permitirán prevenir el riesgo de suicidio en adolescentes chilenos y colombianos mediante la evaluación de estas distorsiones cognitivas, las que también podrán guiar intervenciones psicoterapéuticas reduciendo significativamente la severidad y riesgo de cometer suicidio.

Palabras clave: Distorsiones cognitivas, Riesgo de suicidio, Adolescentes chilenos, Adolescentes colombianos.

Abstract

Objective: This paper aims to determine the relationship between cognitive distortions and risk for suicidal behaviors considering Chilean and Colombian adolescents as sample.

Method: A descriptive-correlational, non-experimental and transversal study was conducted. The risk of suicide was assessed with screening scale of suicidality (scale of Okasha) and the cognitive distortions were determined through Automatic Thoughts Inventory by Ruiz and Lujan. Both of the tests were performed in Chilean ( $n=200$; from Alma Mater School) and Colombian adolescents as sample $(n=200$; educational institution Santa Librada). These adolescents were between 13-18 years-old. This sample was selected through non-probabilistic intentional sample procedure, and the individuals were divided in two groups: one of them are at risk of suicide and the other without suicidal risk (control). A descriptive analysis of

Cómo citar este artículo (APA):

Victoria Álvarez, L., Ayala Moreno, N. Y. \& Bascuñán Cisternas, R. (2019). Las distorsiones cognitivas y el riesgo de suicidio en una muestra de adolescentes chilenos y colombianos: un estudio descriptivo-correlacional . Psicogente 22(41), 1-22. https://doi.org/10.17081/psico.22.41.3308 
the sample, as well as a comparison of the cognitive distortions between groups was performed. Finally, inferential analysis of data is used to determine the relationship and the intensity between cognitive distortions and the risk for suicidal behaviors.

Results: A significant relationship between the cognitive distortions and the suicide risk was found, and the following presented the highest intensity between Chilean and Colombian individuals: Filtration, Interpretation of Though, Polarized Though and Overgeneralization). Moreover, the relationship between the results in Chilean and Colombian adolescents was positive and strong.

Conclusions: Results of this study will allow to prevent risk for suicidal behaviors in Chilean and Colombian adolescents, assessing these cognitive distortions, which make possible to conduct psychotherapeutic interventions significantly, reducing the risk for suicidal behaviors.

Keywords: Cognitive Distortions, Suicide Risk, Chilean Adolescents, Colombian Adolescents.

\section{INTRODUCCIÓN}

El suicidio, del latín sui, uno mismo, y caedĕre, morir (Durkheim, 1928), es el acto intencional mediante el cual el ser humano causa su propia muerte (Organización Mundial de la Salud, OMS, 2006). Asimismo, el suicidio es considerado un problema de salud pública universal y multifactorial (Sarabia, 2014; Gómez, Calle, Victoria \& Flores, 2015; Mosquera, 2016), representando a nivel mundial casi la mitad de las muertes violentas (OMS, 2014), las que se reportan cada 40 segundos, siendo más frecuente en los hombres, ya que estos tienden a utilizar métodos más letales, sin embargo las mujeres presentan el mayor porcentaje de intentos de suicidio (García et al., 2010) y es la segunda causa de muerte en población que atraviesa los 15 y los 29 años de edad (International Association for Suicide Prevention, IASP, 2016; OMS, 2018).

La adolescencia es la etapa del ciclo vital entre los 10 a los 19 años de edad (OMS, 2017), que se caracteriza por la plena vitalidad y vigor (Arias \& Blanco, 2010; Pfeiffer, 2002, citado por Almonte \& Montt, 2012), de acuerdo con la OMS (2001) a nivel mundial se reporta un incremento de suicidios en adolescentes de 15 a 19 años de edad. Sin embargo, para el 2009 se observó una disminución a nivel europeo en las tasas de suicidio para este grupo de edad, no así para los países de Sur América (Kolves \& De Leo, 2016). En países como Chile y Colombia, la mayoría de los suicidios se presentan entre adolescentes que atraviesan los 15 y los 20 años de edad (Instituto Nacional de Medicina Legal y Ciencias Forenses, INMLCF, 2013; Ministerio de Salud y Protección Social, MINSALUD, 2013; Ministerio de Salud del Gobierno de Chile, MINSAL, 2013; Quinlan-Davidson, Sanhueza, Espinosa, Escamilla-Cejudo \& Maddaleno, 2014). Además, según la Organización para la Cooperación y el Desarrollo Económico (OCDE, 2013; Echávarri, Maino, Fischman, Morales \& 
Barros, 2015; Roh, Jung, \& Hong, 2018) Chile se posiciona entre los cinco países con mayor crecimiento en las tasas de suicidio.

Por otra parte, el suicidio es un fenómeno multifactorial, y su prevención se puede reforzar mediante la implementación de la evaluación del riesgo de suicidio (Wasserman, Cheng, \& Jiang, 2005; Gutiérrez, Contreras \& Orozco, 2006; Piedrahita, Paz \& Romero, 2012; Echeburúa, 2015). Según Gómez (2012), el riesgo de suicidio es la probabilidad de desarrollar conductas que atentan intencionalmente contra la vida, con resultado de muerte. Este se clasifica en leve, moderado y severo, y se constituye mediante un proceso que involucra tres fases: el deseo suicida, la idea suicida, y el acto suicida (Bryan \& Rudd, 2006; Nizama, 2011). Por lo tanto, para que se ejecute un suicidio, previamente se activa cierto tipo de pensamientos que propician la aparición de conductas autolesivas (Servicio Andaluz de Salud, 2010). De esta forma, las habilidades autopreservadoras pueden debilitarse frente a estresores ambientales, conduciendo a un mayor riesgo de suicidio.

Estos tipos de pensamientos surgen de esquemas cognitivos, los que se encargan de organizar la información que proviene del ambiente (Beck, 1995). Si bien, esta operación se ejecuta de forma automática, como atajo que facilita el procesamiento de información, también pueden producir errores en su interpretación, generándose así las denominadas distorsiones cognitivas.

Las distorsiones cognitivas consisten en la predisposición para procesar información de acuerdo con los códigos contenidos en los esquemas cognitivos (Beck, 1970, citado por Ruiz, Díaz \& Villalobos, 2012). Inicialmente Beck (1967) propone la existencia de seis distorsiones cognitivas, tales como la Inferencia Arbitraria, Abstracción Selectiva, Generalización, Maximización/ Minimización, Personalización y Pensamiento Absolutista o Dicotómico. Después Beck, Rush, Shaw \& Emery $(1979,1983)$ modifican este listado cambiando algunos nombres y ampliándolo a 11 distorsiones cognitivas: Pensamiento de Todo o Nada, Sobregeneralización, Descontar lo Positivo, Saltar a las Conclusiones, Leer la mente, Adivinación, Magnificar/Minimizar, Razonamiento Emocional, Declaraciones de Debería, Etiquetar y Culpabilidad Inapropiada.

Posteriormente, Mckay, Davis y Fanning (1988) construyen un listado de 15 tipos de distorsiones cognitivas: 
Filtraje: este término es el equivalente a la Abstracción Selectiva de Beck (1967). Mckay et al. (1988) lo definen como una visión de túnel, donde se perciben solo ciertos aspectos de un acontecimiento, sesgando la interpretación del contexto.

Pensamientos Polarizados: cierta inclinación a predecir conclusiones extremistas, con posibilidades opuestas, sin tener evidencia de ello (Mckay et al., 1988). Este es el equivalente a los Pensamientos Dicotómicos o Absolutistas de Beck (1967).

Sobregeneralización: para Mckay et al. (1988) esta distorsión del pensamiento equivaldría a la Generalización Excesiva de Beck (1967) y hace referencia a las conclusiones que surgen de un solo hecho en particular, desplazándose como explicación a otras situaciones aisladas, sin evidencia que lo soporte.

Interpretación del Pensamiento: según Mckay et al. (1988), hace referencia a las conclusiones acerca de los sentimientos e intenciones de otros, sin evidencia alguna de ello.

Visión Catastrófica: de acuerdo con Mckay et al. (1988), consiste en la predisposición a extraer posibles conclusiones catastróficas, sin que existan datos reales que lo evidencien.

Personalización: es la misma distorsión cognitiva que describe Beck (1967) y hace referencia a la atribución o culpabilizarse de hechos ocurridos en el entorno, sin evidencia suficiente (Mckay et al., 1988).

Falacia de Control: Mckay et al. (1988) lo describen como el excesivo control que se ejerce sobre diversos acontecimientos, oscilando entre creencias de alto control o extremos de ningún control.

Falacia de Justicia: percepción de injusticia a todos los hechos que no coincidan con las expectativas o que estén en contra de los deseos propios (Mckay et al., 1988).

Razonamiento Emocional: para Mckay et al. (1988) en esta distorsión, los pensamientos están determinados por el estado afectivo intenso del momento. 
Falacia de Cambio: de acuerdo con Mckay et al. (1988), en esta distorsión los cambios están sujetos a las acciones que ejecuten otros, ya que estos parecen ser los que satisfacen las necesidades propias.

Etiquetas Globales: consiste en el etiquetamiento erróneo y generalizado a hechos del entorno y a otras personas, generando una visión inflexible del mundo (Mckay et al., 1988).

Culpabilidad: según Mckay et al. (1988) esta distorsión surge al atribuirse responsabilidades o acontecimientos a sí mismo o a otros, sin que existe evidencia que lo soporte.

Los Debería: se caracterizan por el desarrollo de normas rígidas y autoexigentes sobre cómo deberían ser las cosas (Mckay et al., 1988).

Falacia de Razón: Mckay et al. (1988) afirman que este tipo de pensamiento ocurre cuando hay una tendencia a probar y reafirmar convicciones propias, frente a situaciones en que se cree tener la razón, sin importar otro tipo de argumentación.

Falacia de Recompensa Divina: consiste en la búsqueda de recompensa divina a partir de comportamientos percibidos como correctos y que aparentemente son merecedores de este premio divino (Mckay et al., 1988).

Las distorsiones cognitivas más frecuentes al comienzo de la adolescencia son el Absolutismo y el Tremendismo y en una fase más tardía la Inferencia Arbitraria y el Razonamiento Emocional (Rodríguez-Testal, Carrasco, Del Barrio \& Catalán, 2002). Las distorsiones cognitivas más típicas en adolescentes con conductas agresivas son las auto-sirvientes, tales como el egocentrismo, culpar a los demás y asumir lo peor y en adolescentes con problemas de ansiedad y depresión, son las auto-humillantes, como la Personalización, Generalización, Abstracción Selectiva y Catastrofismo (Peña \& Andreu, 2012).

De acuerdo con la Tríada Cognitiva de Beck, existe una estructura cognitiva que propicia la activación de estos esquemas disfuncionales, lo que se manifiesta en la percepción de un yo defectuoso, un mundo cruel y un futuro sin esperanza (Ruiz et al., 2012; Toro, 2013). Es así que, las distorsiones cognitivas, al alterar la percepción de esta tríada, podrían estar implicadas en el riesgo de suicidio. 
Asimismo, estas distorsiones cognitivas son un agravante de la alteración emocional (Beck, 1995; Rodríguez -Testal et al, 2002; Gómez, Silva \& Amon, 2017). La alteración en factores como las emociones y la calidad de vida tienen un impacto directo en el riesgo de suicidio en adolescentes (Shain \& Committee On, 2016; Balazs et al., 2018). Así, experiencias de pérdida o de fracaso que se ven afectadas en su interpretación, centrando la atención en aspectos negativos, generan la mantención de estados depresivos y el aumento del riesgo de suicidio.

Las distorsiones cognitivas comparten como característica principal un sesgo en la información procesada, que altera el comportamiento (Gómez, 2012). El estudio de estas distorsiones cognitivas permite la descripción de cierta rigidez cognitiva en adolescentes, que disminuye el despliegue de habilidades adaptativas y de resolución de problemas. Así, el objetivo de este estudio es determinar la relación entre distorsiones cognitivas y el riesgo de suicidio en una muestra de adolescentes chilenos y colombianos.

\section{MÉTODO}

\subsection{Diseño}

Este es un estudio descriptivo-correlacional, de corte no experimental y transversal (Hernández, Fernández \& Baptista, 2014).

\subsection{Participantes}

El tamaño de la muestra fue de 400 adolescentes (hombres y mujeres) entre los 13 y 18 años de edad, de los cuales 200 son estudiantes de nacionalidad colombiana de la institución educativa Santa Librada, de la ciudad de Cali (Colombia) y los otros 200 son estudiantes de nacionalidad chilena del Colegio Alma Mater, de Santiago de Chile (Chile).

\subsubsection{Procedimiento de selección}

El número de participantes se estableció por medio de un muestreo no probabilístico, de tipo intencional, en el cual se tomaron en cuenta aquellos adolescentes que se encontraban disponibles en las instituciones educativas donde se realizan las prácticas profesionales de la Universidad Central de Chile y la Universidad Cooperativa de Colombia.

La muestra se compone así: 99 participantes de género femenino y 101 participantes de género masculino en Chile, y 137 participantes de género 
femenino más 63 participantes de género masculino en Colombia. Para el análisis de los datos se conformaron dos grupos: el grupo sin riesgo de suicidio o control (chilenos y colombianos) y el grupo con riesgo de suicido (chilenos y colombianos).

\subsubsection{Consideraciones éticas}

Este estudio contó con la aprobación y autorización del Comité de Investigaciones de la Universidad Cooperativa de Colombia, sede Cali, que vela por el cumplimiento de los requisitos éticos y legales que rigen la investigación científica.

Puesto que la muestra de los participantes colaboró de forma voluntaria y anónima, no se recolectó información respecto a su identificación. Por tal razón, y siguiendo los lineamientos éticos, una vez establecida la población en potencial riesgo (grupo con riesgo de suicidio en adolescentes chilenos y colombianos) se organizó la información para ser compartida a las instituciones educativas respectivas, con el fin de retroalimentarles acerca de los resultados de este estudio, además de ofrecer como plan de contención acuerdos colaborativos con los Centros de Atención Psicológica de la Universidad Central de Chile y la Universidad Cooperativa de Colombia, donde practicantes de Psicología aumenten su participación en los servicios escolares para la individualización y atención de los casos y de ser necesario, su posterior remisión a dichos centros o a los servicios de salud que correspondan.

\subsection{Instrumentos}

\subsubsection{La escala de Suicidalidad de Okasha}

Detecta el riesgo de suicidio en adolescentes, sin la necesidad de un diagnóstico psiquiátrico previo a su aplicación. La escala está validada en adolescentes chilenos con un coeficiente Alfa de Cronbach de 0,89 (Salvo, Melipillán \& Castro, 2009) y ha sido utilizada en población adolescente de países como México (Perales-Blum \& Loredo, 2015), Perú (Gutiérrez \& Mora, 2017) y Ecuador (Pulla, 2017). Asimismo, el Alfa de Cronbach realizado en este estudio, indica que el coeficiente de fiabilidad de esta escala en adolescentes colombianos es de 0,851 , y en adolescentes chilenos de 0,871 , tal como se observa en la Tabla 1.

La escala está compuesta por cuatro ítems, los cuales evalúan ideación suicida e intento de suicidio (Salvo et al., 2009). La calificación se realiza por 
medio de una escala tipo Likert de 0 a 3 . El resultado de los primeros tres ítems arroja el subpuntaje de ideación suicida y el último ítem conforma el subpuntaje de intento de suicidio. La suma de ambos subpuntajes llega a oscilar entre 0 y 12 puntos, donde el puntaje de corte es igual o mayor a 5, es decir, que a mayor puntuación de 5 , se considera un mayor riesgo de suicidio.

\subsubsection{El inventario de pensamientos automáticos de Ruiz y Luján (1991)}

Se caracteriza por ser una escala autoaplicada para identificar distorsiones cognitivas. De acuerdo con Guevara (2015), el coeficiente de Kaiser-MeyerOlkin (=85) señala una interrelación entre los ítems apropiada para ejecutar un análisis factorial, junto con la prueba de esfericidad de Barlett con valores 3093,004 (df: 153; sig =000). Esta escala fue empleada en población adolescente en países como Colombia (Lemos, Jaller, Gonzales, Díaz \& De la Osa, 2015), Perú (Pérez \& Rosario, 2017) y Ecuador (Guajala \& Paredes, 2015). Asimismo, el Alfa de Cronbach realizado en este estudio, indica que el coeficiente de fiabilidad de este instrumento en adolescentes chilenos es de 0,939 y en los adolescentes colombianos de 0,951, tal como lo describe la Tabla 1.

La escala está compuesta por 45 ítems, los cuales evalúan 15 distorsiones cognitivas, tales como Abstracción Selectiva, Pensamiento Polarizado, Sobregeneralización, Interpretación de Pensamiento, Visión Catastrófica, Personalización, Falacia de Control, Falacia de Justicia, Falacia de Cambio, Razonamiento Emocional, Etiquetas Globales, Culpabilidad, los Deberías, Falacia Razón y Falacia de Recompensa Divina (Moyano, Furlan \& Piemontesi, 2011).

Estas distorsiones cognitivas son evaluadas a partir de su frecuencia de aparición bajo una calificación tipo Likert de 0 a 3. La calificación surge de la sumatoria del puntaje que arroja los ítems correspondientes a cada pensamiento automático, donde una puntuación de 2 o más indica que ese pensamiento genera molestia y una puntuación de 6 o más indica cierta tendencia a sufrir por aquella forma de procesar información.

Tabla 1.

Estadísticas de fiabilidad de la Escala de Suicidalidad de Okasha y del Inventario de Pensamientos Automáticos de Ruiz y Luján para adolescentes chilenos y colombianos

\begin{tabular}{|c|c|c|c|c|c|}
\hline \multicolumn{3}{|c|}{ ADOLESCENTES CHILENOS } & \multicolumn{3}{|c|}{ ADOLESCENTES COLOMBIANOS } \\
\hline $\begin{array}{c}\text { ALFA DE } \\
\text { CRONBACH }\end{array}$ & $\begin{array}{c}\text { N DE } \\
\text { ELEMENTOS }\end{array}$ & PORCENTAJE & $\begin{array}{c}\text { ALFA DE } \\
\text { CRONBACH }\end{array}$ & $\begin{array}{c}\text { N DE } \\
\text { ELEMENTOS }\end{array}$ & PORCENTAJE \\
\hline
\end{tabular}




\begin{tabular}{lllllll}
\hline $\begin{array}{c}\text { Escala de Suicidali- } \\
\text { dad de Okasha }\end{array}$ & 0,871 & 4 & $87,12 \%$ & 0,851 & 4 & $85,06 \%$ \\
$\begin{array}{c}\text { Inventario de } \\
\begin{array}{c}\text { Pensamientos } \\
\text { Automáticos }\end{array}\end{array}$ & 0,939 & 45 & $93,93 \%$ & 0,951 & 45 & $95,13 \%$ \\
\hline
\end{tabular}

\subsection{Procedimiento y análisis de los datos}

En cuanto al trabajo de campo, se realizó una sesión por país para la recolección de los datos. Respecto al análisis de los datos, se construyó una matriz de datos en Microsoft Excel 2013, la cual se exportó al programa estadístico Statistical Package for the Social Sciences (SPSS 23). El análisis estadístico para identificar la relación entre las variables distorsiones cognitivas y riesgo de suicidio fue de carácter no paramétrico, ya que estas variables son ordinales, reducidas a categóricas y de distribución libre.

\subsubsection{Análisis descriptivo-comparativo}

Para realizar el análisis de estadística descriptiva, se aplicó la distribución de frecuencias en cada una de las variables del estudio. Además, se describió la distribución de las distorsiones cognitivas en el grupo sin riesgo de suicidio y en el grupo con riesgo de suicidio.

\subsubsection{Análisis inferencial}

Para determinar la normalidad en los datos, se empleó la prueba de hipótesis estadística Kolmogorov-Smirnov. Dado que la distribución de los datos no es normal, se aplicó la prueba estadística no paramétrica Chi cuadrado $\left(\chi^{2}\right)$, la que indica si existe relación entre las distorsiones cognitivas y el riesgo de suicidio. Además, para identificar la intensidad de la relación entre dichas variables se utilizó la prueba $V$ de Cramer. Por último, se aplicó el coeficiente de correlación de Spearman para identificar la correlación entre los resultados de los participantes chilenos y colombianos.

Por otra parte, para la prueba estadística Chi cuadrado $\left(\chi^{2}\right)$, se supuso un nivel de significancia de $\alpha=0.05$ y los valores que determinaron el límite del grado de aceptación de la hipótesis, de acuerdo con el nivel de significancia y los grados de libertad (gl) son los siguientes:

Tabla 2.

Criterios de distribución del $\chi^{2}$ para determinar el límite del grado de aceptación de la 
hipótesis

Criterios de distribución del $\chi^{2}(\alpha=0,05)$

GRADO DE LIBERTAD (GL)

Por lo tanto, las interpretaciones del Chi cuadrado $\left(\chi^{2}\right)$ se hicieron bajo los siguientes parámetros:

- Hipótesis nula (H0): no existe relación entre las distorsiones cognitivas y el riesgo de suicidio.

- Hipótesis alternativa (H1): existe relación entre las distorsiones cognitivas y el riesgo de suicidio.

- Valores de $\chi^{2}$ menores al límite de aceptación de la hipótesis: se acepta la HO.

- Valores de $\chi^{2}$ mayores al límite de aceptación de la hipótesis: se rechaza la $\mathrm{HO}$.

El coeficiente de correlación de Spearman, se evaluó bajo los siguientes criterios:

- Una relación baja a los valores inferiores a 0,2.

- Una relación media a los valores iguales o mayores que 0,2.

- Una relación alta a los valores superiores o iguales a 0,3.

\section{RESULTADOS}

\subsection{Análisis descriptivo de la muestra}

De los 400 participantes, se obtuvo la muestra completa (200 en Chile y 200 en Colombia). En Chile, el $49 \%$ fueron mujeres y el $51 \%$ hombres, mientras en Colombia el $68 \%$ fueron mujeres y el $32 \%$ fueron hombres. La media de la edad fue de 15,69 años (D.S=1,313) con una edad mínima de 13 años y una máxima de 18 años.

De acuerdo con la prueba de Suicidalidad de Okasha, la muestra se dividió en los siguientes grupos: El grupo sin riesgo de suicidio corresponde a 236 participantes (129 chilenos y 107 colombianos). De este grupo, el 37\% 
corresponde a mujeres y el $63 \%$ a hombres en Chile, en tanto en Colombia se identificó un $64 \%$ y $36 \%$ para mujeres y hombres, respectivamente. Por otro lado, el grupo con riesgo de suicidio incluyó 164 participantes, de los cuales 71 fueron chilenos ( $72 \%$ mujeres y $28 \%$ hombres) y 93 colombianos (74\% mujeres y $26 \%$ hombres), es así como las mujeres reportan el mayor porcentaje con riesgo de suicidio tanto en los adolescentes chilenos como colombianos (Tabla 3).

Tabla 3.

Estadísticas descriptivas de las variables demográficas

\begin{tabular}{|c|c|c|c|c|c|c|c|}
\hline \multirow{2}{*}{ VARIABLE } & & \multicolumn{2}{|c|}{ CHILE } & \multicolumn{2}{|c|}{ COLOMBIA } & \multirow{2}{*}{$\begin{array}{l}\text { FRECUENCIA } \\
\text { ACUMULADA }\end{array}$} & \multirow{2}{*}{$\begin{array}{l}\text { PORCENTAJE } \\
\text { ACUMULADO }\end{array}$} \\
\hline & & FRECUENCIA & PORCENTAJE & FRECUENCIA & PORCENTAJE & & \\
\hline \multirow{6}{*}{ Edad } & 13 años & 18 & 9,0 & 9 & 4,5 & 27 & 13,5 \\
\hline & 14 años & 34 & 17,0 & 16 & 8,0 & 50 & 25,0 \\
\hline & 15 años & 50 & 25,0 & 36 & 18,0 & 86 & 43,0 \\
\hline & 16 años & 53 & 26,5 & 68 & 34,0 & 121 & 60,5 \\
\hline & 17 años & 35 & 17,5 & 54 & 27,0 & 89 & 44,5 \\
\hline & 18 años & 10 & 5,0 & 17 & 8,5 & 27 & 13,5 \\
\hline \multirow{2}{*}{ Género } & Femenino & 99 & 49,5 & 137 & 68,5 & 236 & 118,0 \\
\hline & Masculino & 101 & 50,5 & 63 & 31,5 & 164 & 82,0 \\
\hline \multirow{2}{*}{$\begin{array}{l}\text { Con Riesgo de } \\
\text { Suicidio }\end{array}$} & Femenino & 51 & $72 \%$ & 69 & $74 \%$ & 120 & 146,0 \\
\hline & Masculino & 20 & $28 \%$ & 24 & $26 \%$ & 44 & 54,0 \\
\hline \multirow{2}{*}{$\begin{array}{l}\text { Sin Riesgo de } \\
\text { Suicidio }\end{array}$} & Femenino & 48 & $37 \%$ & 68 & $64 \%$ & 116 & 101,0 \\
\hline & Masculino & 81 & $63 \%$ & 39 & $36 \%$ & 120 & 99,0 \\
\hline
\end{tabular}

\subsection{Análisis comparativo de los grupos}

Como demuestra la Tabla 4, la distorsión cognitiva Falacia de Recompensa Divina es la más frecuente en ambos grupos (con riesgo de suicidio y sin riesgo de suicidio) tanto para los participantes chilenos como colombianos. Además, en el grupo con riesgo de suicidio, las 15 distorsiones cognitivas evaluadas se repiten 694 veces (288 en participantes chilenos y 406 en participantes colombianos), en contraste con el grupo sin riesgo de suicidio donde solo se repiten 247 veces, repartidas en 150 para chilenos y 97 para colombianos. Por lo tanto, la mayor frecuencia de distorsiones cognitivas se encuentra en 
el grupo con riesgo de suicidio, siendo los participantes colombianos los que reportan mayor cantidad de distorsiones cognitivas en dicho grupo.

También se señala en la Tabla 4 que las distorsiones cognitivas (organizadas de mayor a menor frecuencia) Filtraje, Interpretación del Pensamiento, Sobregeneralización y Pensamiento Polarizado presentan mayor frecuencia tanto en chilenos como colombianos del grupo con riesgo de suicidio con respecto a los participantes del grupo sin riesgo de suicidio, donde reportan menor frecuencia y mayores diferencias entre chilenos y colombianos.

Tabla 4.

Comparación de la frecuencia de distorsiones cognitivas entre los grupos

\begin{tabular}{|c|c|c|c|c|c|c|c|}
\hline \multicolumn{4}{|c|}{ CON RIESGO DE SUICIDIO } & \multicolumn{4}{|c|}{ SIN RIESGO DE SUICIDIO } \\
\hline \multirow{2}{*}{$\begin{array}{c}\text { DISTOR- } \\
\text { SIONES } \\
\text { COGNITIVAS }\end{array}$} & \multirow{2}{*}{$\begin{array}{l}\text { CHILE } \\
\text { N DE ELE- } \\
\text { MENTOS }\end{array}$} & \multirow{2}{*}{$\begin{array}{c}\text { DISTOR- } \\
\text { SIONES } \\
\text { COGNITIVAS }\end{array}$} & \multirow{2}{*}{$\begin{array}{c}\text { COLOMBIA } \\
\text { N DE } \\
\text { ELEMENTOS }\end{array}$} & \multirow{2}{*}{$\begin{array}{l}\text { DISTORSIONES } \\
\text { COGNITIVAS }\end{array}$} & \multirow{2}{*}{$\begin{array}{l}\text { CHILE } \\
\text { N DE ELE- } \\
\text { MENTOS }\end{array}$} & \multirow{2}{*}{$\begin{array}{l}\text { DISTORSIONES } \\
\text { COGNITIVAS }\end{array}$} & \multirow{2}{*}{$\begin{array}{c}\text { COLOMBIA } \\
\text { NDE } \\
\text { ELEMENTOS }\end{array}$} \\
\hline & & & & & & & \\
\hline $\begin{array}{l}\text { Falacia de } \\
\text { Recompensa } \\
\text { Divina }\end{array}$ & 45 & $\begin{array}{l}\text { Falacia de } \\
\text { Recompensa } \\
\text { Divina }\end{array}$ & 93 & $\begin{array}{l}\text { Falacia de Re- } \\
\text { compensa Divina }\end{array}$ & 70 & $\begin{array}{c}\text { Falacia de Recompensa } \\
\text { Divina }\end{array}$ & 38 \\
\hline Filtraje & 28 & Filtraje & 38 & Los Debería & 17 & Los Debería & 8 \\
\hline Los Debería & 24 & Los Debería & 30 & Falacia de Razón & 14 & Filtraje & 8 \\
\hline $\begin{array}{c}\text { Interpre- } \\
\text { tación del } \\
\text { pensamiento }\end{array}$ & 24 & $\begin{array}{c}\text { Interpre- } \\
\text { tación de } \\
\text { pensamiento }\end{array}$ & 26 & Falacia de Justicia & 7 & Falacia de Justicia & 7 \\
\hline $\begin{array}{l}\text { Falacia de } \\
\text { Razón }\end{array}$ & 22 & $\begin{array}{l}\text { Falacia de } \\
\text { Razón }\end{array}$ & 25 & $\begin{array}{l}\text { Falacia de } \\
\text { Cambio }\end{array}$ & 7 & Falacia de Razón & 7 \\
\hline $\begin{array}{l}\text { Falacia de } \\
\text { Justicia }\end{array}$ & 21 & $\begin{array}{l}\text { Sobregenerali- } \\
\text { zación }\end{array}$ & 25 & $\begin{array}{c}\text { Visión } \\
\text { Catastrófica }\end{array}$ & 7 & Falacia de Cambio & 6 \\
\hline $\begin{array}{l}\text { Sobregenerali- } \\
\text { zación }\end{array}$ & 19 & $\begin{array}{l}\text { Etiquetas } \\
\text { Globales }\end{array}$ & 24 & Filtraje & 5 & Etiquetas Globales & 4 \\
\hline $\begin{array}{l}\text { Pensamiento } \\
\text { Polarizado }\end{array}$ & 18 & $\begin{array}{l}\text { Pensamiento } \\
\text { Polarizado }\end{array}$ & 24 & $\begin{array}{l}\text { Interpretación de } \\
\text { pensamiento }\end{array}$ & 5 & Visión Catastrófica & 3 \\
\hline $\begin{array}{l}\text { Visión } \\
\text { Catastrófica }\end{array}$ & 17 & $\begin{array}{c}\text { Visión } \\
\text { Catastrófica }\end{array}$ & 22 & $\begin{array}{l}\text { Etiquetas } \\
\text { Globales }\end{array}$ & 4 & Culpabilidad & 3 \\
\hline $\begin{array}{l}\text { Etiquetas } \\
\text { Globales }\end{array}$ & 14 & $\begin{array}{l}\text { Falacia de } \\
\text { Justicia }\end{array}$ & 19 & $\begin{array}{l}\text { Falacia de } \\
\text { Control }\end{array}$ & 4 & $\begin{array}{l}\text { Interpretación del } \\
\text { Pensamiento }\end{array}$ & 3 \\
\hline $\begin{array}{l}\text { Falacia de } \\
\text { Control }\end{array}$ & 14 & $\begin{array}{l}\text { Falacia de } \\
\text { Control }\end{array}$ & 17 & Culpabilidad & 3 & Sobregeneralización & 3 \\
\hline $\begin{array}{c}\text { Falacia de } \\
\text { Cambio }\end{array}$ & 14 & $\begin{array}{l}\text { Falacia de } \\
\text { Cambio }\end{array}$ & 17 & $\begin{array}{c}\text { Razonamiento } \\
\text { Emocional }\end{array}$ & 3 & $\begin{array}{l}\text { Pensamiento } \\
\text { Polarizado }\end{array}$ & 3 \\
\hline Culpabilidad & 13 & Personalización & 17 & Personalización & 2 & Personalización & 2 \\
\hline
\end{tabular}




\begin{tabular}{|c|c|c|c|c|c|c|c|}
\hline $\begin{array}{c}\text { Razonamiento } \\
\text { Emocional }\end{array}$ & 8 & Culpabilidad & 16 & $\begin{array}{c}\text { Sobregenerali- } \\
\text { zación }\end{array}$ & 2 & Falacia de Control & 1 \\
\hline Personalización & 7 & $\begin{array}{l}\text { Razonamiento } \\
\text { Emocional }\end{array}$ & 13 & $\begin{array}{l}\text { Pensamiento } \\
\text { Polarizado }\end{array}$ & 0 & $\begin{array}{c}\text { Razonamiento } \\
\text { Emocional }\end{array}$ & 1 \\
\hline
\end{tabular}

En la Tabla 5 se evidencia que los datos en la muestra completa no presentan distribución normal $(\alpha<0,05)$. Además, de las 15 distorsiones cognitivas evaluadas, la correspondiente a Pensamiento Polarizado no se identificó en ninguno de los participantes chilenos del grupo sin riesgo de suicidio (Ver Tablas 4 y 5 ).

Tabla 5.

Prueba de normalidad para los datos

\begin{tabular}{|c|c|c|c|c|c|c|c|c|c|c|c|c|}
\hline \multicolumn{13}{|c|}{ PRUEBA DE NORMALIDAD KOLMOGOROV-SMIRNOV } \\
\hline \multirow{3}{*}{$\begin{array}{l}\text { DISTORSIONES } \\
\text { COGNITIVAS }\end{array}$} & \multicolumn{6}{|c|}{ CHILE } & \multicolumn{6}{|c|}{ COLOMBIA } \\
\hline & \multicolumn{3}{|c|}{$\begin{array}{l}\text { SIN RIESGO DE } \\
\text { SUICIDIO }\end{array}$} & \multicolumn{3}{|c|}{ CON RIESGO DE SUICIDIO } & \multicolumn{3}{|c|}{$\begin{array}{l}\text { SIN RIESGO DE } \\
\text { SUICIDIO }\end{array}$} & \multicolumn{3}{|c|}{ CON RIESGO DE SUICIDIO } \\
\hline & $\begin{array}{l}\text { ESTADÍS- } \\
\text { TICO }\end{array}$ & GL & SIG. & ESTADÍSTICO & GL & SIG. & $\begin{array}{l}\text { ESTA- } \\
\text { DÍSTICO }\end{array}$ & GL & SIG. & ESTADÍSTICO & GL & SIG. \\
\hline Filtraje & 0,541 & 129 & 0,000 & 0,394 & 71 & 0,000 & 0,537 & 107 & 0,000 & 0,387 & 93 & 0,000 \\
\hline $\begin{array}{l}\text { Pensamiento } \\
\text { Polarizado }\end{array}$ & * & * & * & 0,465 & 71 & 0,000 & 0,539 & 107 & 0,000 & 0,463 & 93 & 0,000 \\
\hline Sobregeneralización & 0,534 & 129 & 0,000 & 0,458 & 71 & 0,000 & 0,539 & 107 & 0,000 & 0,458 & 93 & 0,000 \\
\hline $\begin{array}{l}\text { Interpretación del } \\
\text { pensamiento }\end{array}$ & 0,541 & 129 & 0,000 & 0,423 & 71 & 0,000 & 0,536 & 107 & 0,000 & 0,453 & 93 & 0,000 \\
\hline Visión Catastrófica & 0,540 & 129 & 0,000 & 0,472 & 71 & 0,000 & 0,539 & 107 & 0,000 & 0,474 & 93 & 0,000 \\
\hline Personalización & 0,534 & 129 & 0,000 & 0,530 & 71 & 0,000 & 0,536 & 107 & 0,000 & 0,498 & 93 & 0,000 \\
\hline Falacia de Control & 0,540 & 129 & 0,000 & 0,491 & 71 & 0,000 & 0,529 & 107 & 0,000 & 0,498 & 93 & 0,000 \\
\hline Falacia de Justicia & 0,540 & 129 & 0,000 & 0,444 & 71 & 0,000 & 0,538 & 107 & 0,000 & 0,498 & 93 & 0,000 \\
\hline $\begin{array}{l}\text { Razonamiento } \\
\text { Emocional }\end{array}$ & 0,538 & 129 & 0,000 & 0,526 & 71 & 0,000 & 0,529 & 107 & 0,000 & 0,516 & 93 & 0,000 \\
\hline Falacia de Cambio & 0,540 & 129 & 0,000 & 0,491 & 71 & 0,000 & 0,540 & 107 & 0,000 & 0,498 & 93 & 0,000 \\
\hline Etiquetas Globales & 0,540 & 129 & 0,000 & 0,491 & 71 & 0,000 & 0,540 & 107 & 0,000 & 0,463 & 93 & 0,000 \\
\hline Culpabilidad & 0,538 & 129 & 0,000 & 0,498 & 71 & 0,000 & 0,539 & 107 & 0,000 & 0,503 & 93 & 0,000 \\
\hline Los Debería & 0,519 & 129 & 0,000 & 0,423 & 71 & 0,000 & 0,537 & 107 & 0,000 & 0,431 & 93 & 0,000 \\
\hline Falacia de Razón & 0,527 & 129 & 0,000 & 0,437 & 71 & 0,000 & 0,538 & 107 & 0,000 & 0,458 & 93 & 0,000 \\
\hline $\begin{array}{l}\text { Falacia de Recompen- } \\
\text { sa Divina }\end{array}$ & 0,362 & 129 & 0,000 & 0,409 & 71 & 0,000 & 0,415 & 107 & 0,000 & 0,415 & 93 & 0,000 \\
\hline
\end{tabular}

*Pensamiento Polarizado en Chile es constante en el archivo Sin Riesgo de Suicidio. Se incluirá en cualquier diagrama de cajas generada, pero se omitirá resultado. 0 sujetos Sin Riesgo de Suicidio con esta Distorsión.

\subsection{Análisis inferencial}


Se encontró una relación estadísticamente significativa entre las distorsiones cognitivas y el riesgo de suicidio, excepto para Falacia de Recompensa Divina en los participantes chilenos (Tabla 6).

También, se encontró que las cuatro distorsiones cognitivas más frecuentes (en comparación con el grupo control entre los adolescentes chilenos y colombianos con riesgo de suicidio (Filtraje, Interpretación del Pensamiento, Pensamiento Polarizado y Sobregeneralización; ver Tabla 5) presentan una relación estadísticamente significativa y más intensa con el riesgo de suicidio, con respecto a las otras distorsiones cognitivas (Tabla 6).

Tabla 6.

Análisis inferencial para identificar la relación e intensidad entre las distorsiones cognitivas y el riesgo de suicidio

\begin{tabular}{|c|c|c|c|c|c|c|c|c|c|}
\hline \multicolumn{10}{|c|}{ PRUEBAS ESTADÍSTICAS } \\
\hline \multirow[b]{2}{*}{ DISTORSIONES COGNITIVAS } & \multicolumn{4}{|c|}{ CHILE } & \multirow[b]{2}{*}{ DISTORSIONES COGNITIVAS } & \multicolumn{4}{|c|}{ COLOMBIA } \\
\hline & $X^{2}$ & GL & $\begin{array}{l}\text { VALOR } \\
\text { SIG. }\end{array}$ & $\begin{array}{c}\text { V DE } \\
\text { CRAMER }\end{array}$ & & $x^{2}$ & GL & $\begin{array}{l}\text { VALOR } \\
\text { SIG. }\end{array}$ & $\begin{array}{c}\text { V DE } \\
\text { CRAMER }\end{array}$ \\
\hline Filtraje & 42,033 & 1 & 0,000 & 0,458 & Filtraje & 31,310 & 1 & 0,000 & 0,396 \\
\hline Pensamiento Polarizado & 35,939 & 1 & 0,000 & 0,424 & Interpretación del Pensamiento & 28,125 & 1 & 0,000 & 0,375 \\
\hline Interpretación de Pensamiento & 33,083 & 1 & 0,000 & 0,407 & Sobregeneralización & 23,958 & 1 & 0,000 & 0,346 \\
\hline Sobregeneralización & 30,971 & 1 & 0,000 & 0,394 & Pensamiento Polarizado & 22,545 & 1 & 0,000 & 0,336 \\
\hline Falacia de Justicia & 22,185 & 1 & 0,000 & 0,333 & Etiquetas Globales & 20,125 & 1 & 0,000 & 0,317 \\
\hline Culpabilidad & 15,897 & 1 & 0,000 & 0,282 & Los Debería & 19,854 & 1 & 0,000 & 0,315 \\
\hline Falacia de Control & 15,441 & 1 & 0,000 & 0,278 & Visión Catastrófica & 19,780 & 1 & 0,000 & 0,314 \\
\hline Etiquetas Globales & 15,441 & 1 & 0,000 & 0,278 & Falacia de Control & 18,277 & 1 & 0,000 & 0,302 \\
\hline Visión Catastrófica & 14,870 & 1 & 0,000 & 0,273 & Falacia de Recompensa Divina & 16,747 & 1 & 0,000 & 0,289 \\
\hline Falacia de Razón & 12,576 & 1 & 0,000 & 0,251 & Personalización & 15,585 & 1 & 0,000 & 0,279 \\
\hline Los Debería & 11,953 & 1 & 0,001 & 0,244 & Falacia de Razón & 15,315 & 1 & 0,000 & 0,277 \\
\hline Falacia de Cambio & 9,954 & 1 & 0,002 & 0,223 & Razonamiento Emocional & 13,004 & 1 & 0,000 & 0,255 \\
\hline Personalización & 7,357 & 1 & 0,007 & 0,192 & Culpabilidad & 12,001 & 1 & 0,001 & 0,254 \\
\hline Razonamiento Emocional & 7,045 & 1 & 0,008 & 0,188 & Falacia de Justicia & 8,485 & 1 & 0,004 & 0,206 \\
\hline Falacia de Recompensa Divina & 1,558 & 1 & 0,212 & 0,088 & Falacia de Cambio & 7,811 & 1 & 0,005 & 0,198 \\
\hline
\end{tabular}

La correlación entre los resultados de la muestra de adolescentes chilenos y colombianos es positiva y alta (Rho de Spearman $=0,588$ ), como se demuestra en la Tabla 7.

Tabla 7.

Prueba estadística para determinar correlación entre los resultados de los adolescentes chilenos y colombianos 


\begin{tabular}{|c|c|c|c|c|}
\hline \multicolumn{5}{|c|}{ CORRELACIONES } \\
\hline & & & $\begin{array}{l}\text { DISTORSIONES } \\
\text { COGNITIVAS/RIES- } \\
\text { GO DE } \\
\text { SUICIDIO } \\
\text { CHILE }\end{array}$ & $\begin{array}{c}\text { DISTORSIONES COG- } \\
\text { NITIVAS/RIESGO DE } \\
\text { SUICIDIO } \\
\text { COLOMBIA }\end{array}$ \\
\hline \multirow{6}{*}{ Rho de Spearman } & \multirow{3}{*}{$\begin{array}{l}\text { Distorsiones Cogniti- } \\
\text { vas/Riesgo de Suicidio } \\
\text { Chile }\end{array}$} & Coeficiente de correlación & 1,000 & 0,588 \\
\hline & & Sig. (bilateral) & & 0,021 \\
\hline & & N & 15 & 15 \\
\hline & \multirow{3}{*}{$\begin{array}{l}\text { Distorsiones Cogniti- } \\
\text { vas/Riesgo de Suicidio } \\
\text { Colombia }\end{array}$} & Coeficiente de correlación & 0,588 & 1,000 \\
\hline & & Sig. (bilateral) & 0,021 & \\
\hline & & $\mathrm{N}$ & 15 & 15 \\
\hline
\end{tabular}

\section{DISCUSIONES}

Con el análisis de los resultados obtenidos se comprueba la hipótesis en la cual las distorsiones cognitivas se relacionan con el riesgo de suicidio en una muestra de adolescentes chilenos y colombianos.

Respecto a dicha relación entre las variables de la investigación, se observa que todas las distorsiones cognitivas presentan una relación estadísticamente significativa con el riesgo de suicidio en la muestra de adolescentes chilenos y colombianos, salvo la Falacia de Recompensa Divina en los participantes chilenos, de manera que las distorsiones cognitivas dan cuenta de cierta predisposición para procesar información (Gutiérrez, Ceniceros \& Méndez, 2012), provocando una disminución de las respuestas adaptativas.

De ahí que, en la distribución de frecuencias, el grupo con riesgo de suicidio reporte la mayor cantidad de distorsiones cognitivas. Asimismo, en el grupo con riesgo de suicidio se observa un mayor porcentaje de mujeres tanto en los participantes chilenos como colombianos, lo cual coincide con el estudio de García et al. (2010) donde el género femenino corre mayor riesgo de presentar intentos de suicidio.

También llama la atención que se observen cuatro distorsiones cognitivas (Filtraje, Interpretación del Pensamiento, Pensamiento Polarizado y Sobregeneralización), que además de ser las más frecuentes en los participantes chilenos y colombianos con riesgo de suicidio (en comparación con el grupo control), guardan relación estadísticamente significativa e intensa con el riesgo de suicidio. Por consiguiente, en el grupo de adolescentes con riesgo de suicidio tanto chilenos como colombianos, se presenta una estructura cognitiva que propicia la aparición de sesgos cognitivos similares en el proce- 
samiento de la información (Ruiz et al., 2012), generando cierta vulnerabilidad al momento de responder adaptativamente a situaciones conflictivas.

Estos resultados coinciden con tres de las distorsiones cognitivas más típicas en adolescentes con problemas de ansiedad y depresión según Peña y Andreu (2012): Generalización, Abstracción Selectiva y Catastrofismo (excepto Personalización). Así mismo, en el presente estudio las distorsiones cognitivas más frecuentes y con relación estadísticamente significativa e intensa con el riesgo de suicidio son: Filtraje (equivalente a la Abstracción Selectiva), Pensamiento Polarizado (equivalente a Visión Catastrófica), Sobregeneralización (equivalente a Generalización) e Interpretación del Pensamiento.

Por otra parte, la activación de estos esquemas disfuncionales se manifiesta en las aristas de la Tríada Cognitiva de Beck (1967), es decir, en la percepción alterada de sí mismo, una visión sesgada del mundo y la idea de un futuro desolador (Ruiz et al., 2012; Toro, 2013). En el estudio realizado por Moral y Sirvent (2011) se encontró que dicha tríada cognitiva se presenta en estudiantes de secundaria con ideación y comportamientos suicidas. En este sentido, al rescatar solo aspectos negativos del mundo, del futuro y de sí mismo, estas distorsiones cognitivas constituyen un potencial factor de riesgo para el suicidio en adolescentes.

Para Mckay et al. (1988) el Filtraje es una distorsión cognitiva que está determinada por interpretaciones sesgadas, producto de una visión de túnel. Asimismo, la Interpretación del Pensamiento, según este autor, es una distorsión que se caracteriza por la presencia de conclusiones sobre los sentimientos o intención de otros sin evidencia alguna.

Así pues, en el caso del riesgo de suicidio, pareciera que los adolescentes que presentan estas distorsiones cognitivas (Filtraje e Interpretación del Pensamiento) seleccionan de diversas situaciones solo los aspectos negativos, generando una interpretación sesgada de sí mismos y asumiendo intenciones hostiles de otros, lo que disminuye las posibilidades de enfrentar escenarios conflictivos o el despliegue de habilidades adaptativas. Toro (2013) coincide en que la ideación suicida es gatillada por "cogniciones negativas autorreferentes" (p.93) que propician un procesamiento de información rígido y sesgado empobreciendo la capacidad para resolver problemas. 
En el caso de la Sobregeneralización, de acuerdo con Mckay et al. (1988), se tiende a extraer conclusiones, desplazándolas como explicación a otras situaciones aisladas, por lo cual si en los adolescentes con riesgo de suicidio el escenario parece ser un problema de proporciones, probablemente se espere que siempre sea igual, lo que se traduce en la perspectiva de un futuro sin solución. Igualmente, en el Pensamiento Polarizado, al generarse conclusiones extremistas (Mckay et al., 1988) en los adolescentes con riesgo de suicidio se hace presente la posibilidad de un futuro sin opciones de cambio, lo cual concuerda con el estudio realizado por Gonzales y Hernandez (2012) donde concluyen que la pérdida de motivación y expectativas negativas del futuro son factores predictores del riesgo de suicidio.

Por otra parte, estos resultados permiten ahondar en la comprensión de la alteración emocional durante el procesamiento de información (Beck, 1995, citado por Rodríguez-Testal et al., 2002; Gómez, Silva \& Amon, 2017), alteración que tiene un impacto directo en el riesgo de suicidio en adolescentes (Shain \& Committee On, 2016; Balazs et al., 2018). En este sentido, la activación de dichas distorsiones cognitivas (Filtraje, Interpretación del Pensamiento, Pensamiento Polarizado y Sobregeneralización), generan sesgos en el procesamiento de información, provocando la contención de emociones descompensadoras y desesperanzadoras, lo que podría aumentar el riesgo de suicidio. Asimismo, Gonzales y Hernández (2012) encontraron que durante el proceso psicoterapéutico en jóvenes con riesgo de suicidio se debe trabajar en el adecuado manejo de las emociones y en la reestructuración de pensamientos disfuncionales.

También se confirma una correlación intensa y positiva entre los resultados de los participantes chilenos y colombianos, con una similitud del $59 \%$, lo cual es un resultado relevante, pues indica un nivel relativamente alto de generalidad intercultural. Por esta razón, es posible afirmar que, los adolescentes del grupo con riesgo de suicidio de ambos países presentan una tendencia en los sesgos cognitivos durante el procesamiento de la información, lo que disminuye el despliegue de habilidades adaptativas y de resolución de problemas (Rojas, 2013), por lo que pareciera que las distorsiones cognitivas se presentan como un factor agravante mas no causal del riesgo de suicidio.

Por lo tanto, estas distorsiones cognitivas (Filtraje, Interpretación del Pensamiento, Pensamiento Polarizado y Sobregeneralización) se constituyen como una variable que además de estar relacionadas con el riesgo de suicidio en los participantes de la muestra, pueden ser un factor predictor 
en las intervenciones psicoterapéuticas, así como guiarlas y mejorarlas, pues como lo indica Jara, Serrano, Paz y Urizar (2001) en personas con ideación o conductas suicidas se debe evaluar el funcionamiento cognitivo y emocional, para realizar propuestas de intervención más efectivas.

Por último, este estudio tuvo como objetivo determinar la relación entre las distorsiones cognitivas y el riesgo de suicidio en una muestra de adolescentes, chilenos y colombianos, por lo cual es recomendable determinar en futuras investigaciones la relación con otras variables como el género, la edad, o incluir participantes de otras nacionalidades, de manera que se logre un análisis más completo, además de ampliar la muestra para alcanzar mayor representatividad en la población y por ende, mayor confiabilidad y generalidad de los resultados. Asimismo, determinar los factores de riesgo en el suicidio ayuda a su prevención (Wasserman et al., 2005; Gutiérrez et al., 2006; Piedrahita et al., 2012; Echeburúa, 2015). Por lo tanto, nuestros resultados son relevantes, ya que al establecer los aspectos particulares del funcionamiento cognitivo de una muestra de adolescentes con riesgo de suicidio, se propicia la elaboración de estrategias terapéuticas clínicas más eficientes y preventivas en esta población.

Como se ha sugerido en diferentes investigaciones, la adolescencia se ha transformado en un área fecunda de análisis, toda vez que se ha puesto de manifiesto la importancia de realizar estudios para prevenir la aparición de enfermedades y problemas de salud, así como también promover habilidades y entregar herramientas que le permitan a los adolescentes no solo disminuir la probabilidad de tener problemas de salud, sino también para promover su bienestar y aumentar su calidad de vida. Por consiguiente, los resultados del presente estudio permitirán prevenir con mayor efectividad el riesgo de suicidio en adolescentes chilenos y colombianos, mediante la detección y evaluación de estas distorsiones cognitivas, guiando a su vez las intervenciones psicoterapéuticas en adolescentes, pues dirigirlas a este tipo de distorsiones propiciará la reducción significativa en la severidad y el riesgo de cometer suicidio.

Financiamiento: Artículo derivado del proyecto Distorsiones cognitivas asociadas al riesgo de suicidio en adolescentes de nacionalidad chilena y colombiana, correspondiente al semillero de investigación Cognición y Comportamiento de la Universidad Cooperativa de Colombia, sede Cali.

Agradecimientos: A la Institución Educativa Santa Librada, de Cali (Colombia), al Colegio Alma Mater, de Santiago de Chile (Chile) por apoyar el proceso de 
recolección de información y al docente Luis Gajardo Ibáñez de la Universidad Central de Chile por recomendaciones en el análisis de los datos.

\section{REFERENCIAS}

Almonte, C., \& Montt, M.E. (2012). Psicopatología infantil y de la adolescencia. Segunda edición. Chile: Mediterráneo.

Arias, E., \& Blanco, I. (2010). Una aproximación al entendimiento del suicidio en comunidades rurales y remotas de América Latina. Estudios Sociológicos, 28(82), 185-210. Disponible en: http://www.redalyc.org/articulo.oa?id=59820675008

Balazs, J., Miklosi, M., Halasz, J., Horvath, L. O., Szentivanyi, D., \& Vida, P. (2018). Suicidal Risk, Psychopathology, and Quality of Life in a Clinical Population of Adolescents. Front Psychiatry, 9, 17. https://doi.org/10.3389/fpsyt.2018.00017

Beck, A.T. (1967). Depression: Clinical Experimental and Theoretical Aspects. New York: Harper and Row.

Beck, A.T. (1995). Cognitive therapy: basics and beyond. New York: Guilford Publication.

Beck, A.T, Rush, A.J., Shaw, B.F. \& Emery, G. (1979). Cognitive Therapy of Depression. New York: The Guilford Press.

Beck, A.T, Rush, A.J., Shaw, B.F. \& Emery, G. (1983). Terapia cognitiva de la depresión. Bilbao: Desclée de Brouwer

Bryan, C.J. \& Rudd, M.D. (2006).Advances in the assessment of suicide risk. Journal of Clinical Psychology, 62(2), 185-200. https://doi.rog/10.1002/jclp.20222

Durkheim, E. (1928). El suicidio: Estudio de Sociología. Primera edición. Madrid: Reus S. A.

Echávarri, O., Maino, M.P., Fischman, R., Morales, S., \& Barros, J. (2015). Aumento sostenido del suicidio en Chile: un tema pendiente. Centro de Políticas Públicas, 10(79), 3-14. Disponible en: https://politicaspublicas.uc.cl/wp-content/ uploads/2015/07/N\%C2\%B0-79-Aumento-sostenido-del-suicidio-en-Chile.pdf

Echeburúa, E. (2015). Las múltiples caras del suicidio en la clínica psicológica. Revista Terapia Psicológica, 33(2), 117-126. Disponible en https://scielo.conicyt.cl/pdf/ terpsicol/v33n2/art06.pdf

García, H., Sahagún, J.E., Ruiz, A., Sánchez, G.M., Tirado, J.C., \& González, J.G. (2010). Factores de riesgo, asociados a intento de suicidio, comparando factores de alta y baja letalidad. Revista de salud pública, 12(5), 713-721. Disponible en: https:// www.scielosp.org/article/ssm/content/raw/?resource_ssm_path=/media/ assets/rsap/v12n5/v12n5a02.pdf

Gómez, A. (2012). Evaluación de Riesgo de Suicidio: Enfoque Actualizado. Revista Médica Clínica Las Condes, 23(5), 607-615. Disponible en: https:// www.clinicalascondes.cl/Dev_CLC/media/Imagenes/PDF\%20revista\%20 m\%C3\%A9dica/2012/5\%20sept/11_Dr--Alejandro-Gomez-C.-4.pdf

Gómez, A., Silva, H. \& Amon, R. (2017). Prevención del Suicidio: Teoría y Práctica. Chile: Editorial Mediterráneo.

Gómez, M., Calle, D., Victoria, L. \& Flores, J. (2015). Adolescencia y suicidio: Entre la perspectiva neuropsicológica de Diamond y Psicoevolutiva de Dolto. Realitas, Revista de Ciencias Sociales, Humanas y Arte, 3(1), 37-43. Disponible en: http:// www.academia.edu/14112574/Adolescencia_y_suicidio_Entre_la_perspectiva_ neuropsicol\%C3\%B3gica_de_Diamond_y_Psicoevolutiva_de_Dolto

Gonzales, J. \& Hernández, A. (2012). La desesperanza aprendida y sus predictores en jóvenes: análisis desde el modelo de Beck. Enseñanza e Investigación en Psicología, 
17(2), 313-327. Disponible en: http://www.redalyc.org/pdf/292/29224159015. pdf

Guajala, J.A., \& Paredes, E.B. (2015). Pensamientos distorsionados en las embarazadas adolescentes de 14-18 años de edad y su influencia social y familiar en el Hospital Gineco-Obstetra Isidro Ayora (Tesis de pregrado). Repositorio digital. Universidad Central del Ecuador, Ecuador. Disponible en: http://www.dspace. uce.edu.ec/handle/25000/7153

Guevara, A.J. (2015). Las distorsiones cognitivas y su relación con el riesgo suicida en los internos del Centro de Privación de Libertad de Personas Adultas Ambato (Tesis de pregrado). Repositorio digital. Universidad Técnica de Ambato, Ecuador. Disponible en: http://repo.uta.edu.ec/handle/123456789/12077

Gutiérrez, A.G., Contreras, C.M. \& Orozco, R.C. (2006). El suicidio, conceptos actuales. Salud mental, 29(5), 66-74. Disponible en: http://www.redalyc.org/articulo. oa?id $=58229510$

Gutiérrez, D., Ceniceros D.I. \& Méndez, A. (2012). Cognición y procesos de aprendizaje. México: ReDIE-Anglo.

Gutiérrez, M.V. \& Mora, J.E. (2017). Disfunción familiar y suicidalidad en adolescentes de la Institución Educativa Cristo Rey (Tesis de maestría). Repositorio Institucional. Universidad Privada Antonio Guillermo Urrelo, Perú. Disponible en: http:// repositorio.upagu.edu.pe/bitstream/handle/UPAGU/651/Tesis\%20Maria\%20 Gutierrez\%20-\%20Janine\%20Mora.pdf?sequence=1\&isAllowed=y

Hernández, R., Fernández, C. \& Baptista, M.P. (2014). Metodología de la investigación. Sexta edición. México D.F: McGraw-Hill Interamericana Editores.

Instituto Nacional de Medicina Legal y Ciencias Forenses. (2013). Quitarse la vida cuando esta aún comienza. Boletín epidemiológico trimestral, 6(8), 1-14. Disponible en: http://www.medicinalegal.gov.co/documents/20143/57949/Quitarse+la+vida+cuando+\%C3\%A9sta+a\%C3\%BAn+comienza.pdf

International Association for Suicide Prevention. (2016). News Bulletin. Disponible en: https://www.iasp.info/pdf/newsletters/2016_november.pdf

Jara, A. B., Serrano, F. G., Paz, M., \& Urizar, M. (2001). Aproximación a las tentativas de suicidio en los adolescentes. Cuadernos de Psiquiatría y Psicoterapia del Niño y del Adolescente, 31(32), 115-139. Disponible en: http://www.sepypna.com/ documentos/psiquiatria31_32b.pdf

Kolves, K., \& De Leo, D. (2016). Adolescent Suicide Rates between 1990 and 2009: Analysis of Age Group 15-19 Years Worldwide. J Adolesc Health, 58(1), 69-77. http://doi.org/10.1016/j.jadohealth.2015.09.014

Lemos, M., Jaller, C., González, A. M., Díaz, Z. T. \& De la Ossa, D. (2015). Perfil cognitivo de la dependencia emocional en estudiantes universitarios en Medellín, Colombia. Universitas Psycologica, 11(2), 395-404. Disponible en: http://www. redalyc.org/html/647/64723241004/

Mckay, M., Davis, M. \& Fanning, P. (1988). Técnicas cognitivas para el tratamiento del estrés. Primera edición. España: Martínez Roca S. A.

Ministerio de Salud del Gobierno de Chile. (2013). Programa nacional de prevención del suicidio: Orientaciones para su implementación. Subsecretaría de salud pública, 27, 2-72. Disponible en: https://www.minsalud.gov.co/Paginas/Nueval\%C3\%ADneatelef\%C3\%B3nicagratuitayp\%C3\%A1ginawebparaatenci\%C3\%B3ndeadiccionesysaludmental.aspx

Ministerio de Salud \& Protección Social. (2013). Análisis de Situación de Salud según regiones Colombia. ASIS Nacional, Colombia, 5-252. Disponible en: https://www. 
minsalud.gov.co/Documentos\%20y\%20Publicaciones/An\%C3\%A1lisis\%20de\%20 situaci\%C3\%B3n\%20de\%20salud\%20por\%20regiones.pdf

Moral, M. \& Sirvent, C. (2011). Desórdenes afectivos, crisis de identidad e ideación suicida en adolescentes. International Journal of Psychology and Psychological Therapy, 11(1), 33-56. Disponible en: https://es.scribd.com/ document/113884055/Desordenes-afectivos-crisis-de-identidad-e-ideacion-suicida-en-adolescentes

Mosquera, L. (2016). Conducta suicida en la infancia: una revisión crítica. Revista de Psicología Clínica con niños y adolescentes, 3(1) 9-18. Disponible en: http:// observatorioninez.consejoinfancia.gob.cl/wpcontent/uploads/2017/09/ conducta_suicida.revision_critiapdf

Moyano, M., Furlan, L., \& Piemontesi, S. E. (2011). Análisis factorial exploratorio del inventario de pensamientos automáticos (IPA). III Congreso Internacional de Investigación y Práctica Profesional en Psicología. XVIII Jornadas de Investigación Séptimo Encuentro de Investigadores en Psicología del MERCOSUR. Universidad de Buenos Aires. Argentina. Disponible en: https://www.aacademica.org/000052/928.pdf

Nizama, M. (2011). Suicidio. Revista Peruana de Epidemiología, 15(2), 81-85. Disponible en: http://www.redalyc.org/articulo.oa?id=203122516002

Organización Mundial de la Salud (2001). Prevención del suicidio un instrumento para docentes y demás personal institucional. Disponible en: http://www.who. int/mental_health/media/en/63.pdf?ua=1

Organización Mundial de la Salud (2006). Prevención del suicidio un instrumento para el trabajo. Disponible en: http://www.who.int/mental_health/prevention/ suicide/resource_work_spanish.pdf?ua=1

Organización Mundial de la Salud (2014). Primer informe de la OMS sobre prevención del suicidio. Disponible en: http://www.who.int/mediacentre/news/ releases/2014/suicide-prevention-report/es/

Organización Mundial de la Salud (2017). Adolescentes: riesgos para la salud y soluciones. Disponible en: http://www.who.int/mediacentre/factsheets/fs345/ es/

Organización Mundial de la Salud (2018). Suicidio. Disponible en: http://www.who. int/mediacentre/factsheets/fs398/es/

Organización para la Cooperación y el Desarrollo Económico. (2013). Panorama de Salud: Informe OECD sobre Chile y comparación con países miembros. Ministerio de Salud Gobierno de Chile. 2-37. Disponible en: http://www.minsal.cl/sites/ default/files/INFORME\%200CDE_2013_21\%2011_final.pdf

Peña, M.E., \& Andreu, J.M. (2012). Distorsiones cognitivas: una revisión sobre sus implicaciones en la conducta agresiva y antisocial. Psicopatología Clínica, Legal y Forense, 12, 85-99. Disponible en: http://masterforense.com/pdf/2012/2012art5. pdf

Perales-Blum, M.T.L., \& Loredo, L. (2015). Family dysfunction and suicidality in adolescents with major depression disorder. Salud mental, 38(3), 195-200. http://doi. org/10.17711/SM.0185-3325.2015.027

Pérez, E.R., \& Rosario, F.J. (2017). Propiedades psicométricas del Inventario de Pensamientos Automáticos para estudios vinculados a la conducta antisocial y delictiva en estudiantes de secundaria de instituciones educativas del distrito de Comas. PsiqueMag, 6(1), 183-199. http://ojs.ucvlima.edu.pe/index.php/psiquemag/ article/view/182/99 
Piedrahita, L.E., Paz, K.M., \& Romero, A.M. (2012). Estrategia de intervención para la prevención del suicidio en la adolescencia: la escuela como contexto. Hacia la Promoción de la Salud. 17(2), 136-148. Disponible en: http://vip.ucaldas.edu.co/ promocionsalud/downloads/Revista17(2)_10.pdf

Pulla, E.N. (2017). Autoestima de los adolescentes y su relación con el contexto familiar, escolar y social. Jadán, Gualaceo 2015 (Tesis de posgrado). Repositorio Institucional. Universidad de Cuenca, Ecuador. Disponible en: http://dspace. ucuenca.edu.ec/bitstream/123456789/27112/1/Tesis.pdf

Quinlan-Davidson, M., Sanhueza, A., Espinosa, I., Escamilla-Cejudo, J. A., \& Maddaleno, M. (2014). Suicide among young people in the Americas. J Adolesc Health, 54(3), 262-268. https://doi.org/10.1016/j.jadohealth.2013.07.012

Rodríguez-Testal, J.F., Carrasco, M.A., Del Barrio, V., \& Catalán, Mạ. C. (2002). Errores cognitivos en jóvenes y su relación con la sintomatología depresiva. Anuario de Psicología, 33(3), 409-432. Disponible en: https://idus.us.es/xmlui/bitstream/ handle/11441/43142/Errores\%20cognitivos.pdf?sequence $=1$

Roh, B. R., Jung, E. H., \& Hong, H. J. (2018). A Comparative Study of Suicide Rates among 10-19-Year-Olds in 29 OECD Countries. Psychiatry Investig, 44(2), 14-28. http://doi.org/10.30773/pi.2017.08.02

Rojas, M.E. (2013). Distorsiones cognitivas y conducta agresiva en jóvenes y adolescentes: análisis en muestras comunitarias y de delincuentes (Tesis doctoral). Universidad Complutense de Madrid, España. Disponible en: http://eprints.ucm. es/23510/1/T34887.pdf

Ruiz, J., \& Luján, J. (1991). Manual de Psicoterapia Cognitiva. Inventario de pensamientos automáticos. Disponible en: http://www.psicologiaonline.com/ ESMUbeda/Libros/SentirseMejor/sentirse2.htm

Ruiz, M.A., Díaz, M.I. \& Villalobos, A. (2012). Manual de técnicas de intervención cognitivo conductuales. Bilbao: Desclee De Brouwer.

Salvo, G.L., Melipillán A.R., \& Castro S. A. (2009). Confiabilidad, validez y punto de corte para escala de screening de suicidalidad en adolescentes. Revista chilena de neuro-psiquiatría, 47(1), 16-23. Disponible en: https://dx.doi.org/10.4067/ S0717-92272009000100003

Sarabia, S. (2014). Suicidio. Un problema de Salud Pública. Revista de Neuropsiquiatría, 77(4) 199-200. Disponible en: www.scielo.org.pe/pdf/rnp/v77n4/ a01v77n4.pdf

Servicio Andaluz de Salud (2010). Recomendaciones sobre la detección, prevención e intervención de la conducta suicida. Andalucia: Área de Dirección de Organizaciones Sanitarias.

Shain, B., \& Committee On, A. (2016). Suicide and Suicide Attempts in Adolescents. Pediatrics, 138(1). https://doi.org/10.1542/peds.2016-1420

Toro, R. (2013). Conducta suicida: consideraciones prácticas para la implementación de la terapia cognitiva. Psychologia: Avances de la Disciplina, 7(1), 93-102. Disponible en: http://www.redalyc.org/articulo.oa?id=297226904010

Wasserman, D., Cheng, Q., \& Jiang, G. X. (2005). Global suicide rates among young people aged 15-19. World Psychiatry, 4(2), 114-120. Disponible en: https://www. ncbi.nlm.nih.gov/pmc/articles/PMC1414751/ 